\title{
The Mental Retardation Report Framework
}

\author{
BARRY A. TANNER \\ Detroit Receiving Hospital and University Health Center \\ and Wayne State University, Detroit, Michigan
}

\begin{abstract}
The Mental Retardation Report Framework (MR Framework) assists in reporting the results of psychological evaluations of patients who are either known or suspected to be mentally retarded. The program allows the psychologist to produce the framework of a report in a few minutes, accepting keyboard input for free-form text and mouse clicks for selecting from lists of choices. Input includes the patient's history, the psychologist's observations, an adaptive behavior scale, and an intelligence test. The report may be edited and printed from within the program, or saved to a file that can be loaded into the psychologist's favorite word processor.
\end{abstract}

Working in a teaching hospital, I soon recognized a tension between the testing patient's need for rapid services and the inexperienced, relatively slow trainee's need to learn by doing and to discuss multiple revisions of reports with a supervisor. I also found that many students had difficulty in following my outline for typical reports. Finally, I was increasingly impatient with having to read handwritten and/or poorly organized first drafts of reports. I decided to deal with all three of these issues by programming a report generator that would ensure a consistent organization and presentation of information, assist my students in producing reports more quickly, and reduce the burden on my middle-aged eyes by creating printed reports, even in first draft.

I selected the mental retardation evaluation for the development of a report generator because it had become relatively standardized for us. Our patients required an intelligence scale and an adaptive behavior scale, and we had previously settled on the WAIS-R and the Vineland Adaptive Behavior Scale for this purpose. Looking through old testing files, I determined that a fairly narrow range of information and descriptors was already being used, and I chose to formalize those choices.

I first developed a series of macros within Word 5.0 to produce the report. With the experience gained from that first version, I next wrote a $C$ program that ran under DOS (Tanner, 1993). Then, in order to provide a more flexible and contemporary interface, I decided to rewrite the program in Visual Basic. This allowed for relatively easy inclusion of a menu bar, drop-down menus, multiple windows, a mouse-aware program, true WYSIWYG, multiple fonts, and the ability to save files in several formats, including ASCII and RTF.

The program is organized around screens or data views, similar to the paper forms that a psychologist might complete. Unlike the previous linear version, the MR Framework allows the user to move among data fields accord-

Address correspondence to B. A. Tanner, Psychology 9B, Detroit Receiving Hospital and University Health Center, 4201 St. Antoine, Detroit, MI 48201. ing to preference. The user may proceed through the views in the suggested order-for example, moving from identifying data to referral data-or may choose to follow any sequence of views. The user can move through the data fields in a given view in any sequence, and is able to skip fields that may not be pertinent for a particular patient without leaving obvious gaps in the printed report.

The program begins with the Main View (see Figure 1). The psychologist clicks on the view menu at the top of the screen, which drops down a list of the 10 data views. Once the information has been entered into a data view, the user clicks on the $<\mathrm{OK}>$ button at the bottom of the view to accept it, and then clicks on the $<$ Next $>$ button to move to the next view. The psychologist can move backward by clicking on $<$ Back $>$. Most reports will begin with the Identifying Information View, which collects demographic data. The psychologist will then generally enter information about the referral source and referral question, as well as information about the person who accompanied the patient. The History View (see Figure 2) collects a brief, relevant history for the patient, which is followed by observations of the patient's behavior and scores on the WAIS-R (Figure 3) and the Vineland. The last data view collects and organizes the psychologist's diagnosis, referrals, and recommendations. Clicking on $<$ Main > returns the user to the Main View.

Some items are context sensitive. For example, if the psychologist indicates that there is reason to question the Vineland, additional items are presented so as to identify why the results are questionable. Similarly, the educational items regarding highest age or grade appear only if the patient has attended school, the type of work is presented only if the patient has held paid employment, and a text box for details regarding when the patient was identified as mentally retarded appears only if the psychologist checks "other" (all in Figure 2). The text scrolls to allow more input than will fit within the dimensions of the boxes.

Clicking on < Report > at the top of the Main View produces a report that incorporates the information just entered (see Figure 4). That report may be edited and 
printed within the Framework and saved to disk. The Framework provides considerable text-processing capabilities, as well as the option of saving the file in formats that many commercial word processors can read.

The American Psychological Association's guidelines for developers of interpretive programs states that adequate information about the system should be made available to allow peer review (American Psychological Association, 1986). Statements in the Framework are based upon Silverstein's (1982) criteria for reliable differences in age-scaled subtest scores for the WAIS-R and from the manual (Sparrow, Balla, \& Cicchetti, 1984) for the Vineland.

The MR Framework was written in Visual Basic 3.0 and uses HighEdit 1.10, a custom control, to provide word-processing capability. The program requires Windows 3.0 or later and requires $716 \mathrm{~K}$ of available disk space with the runtime library.

Psychologists who are interested in using the Framework should send me a letter stating that they are quali-

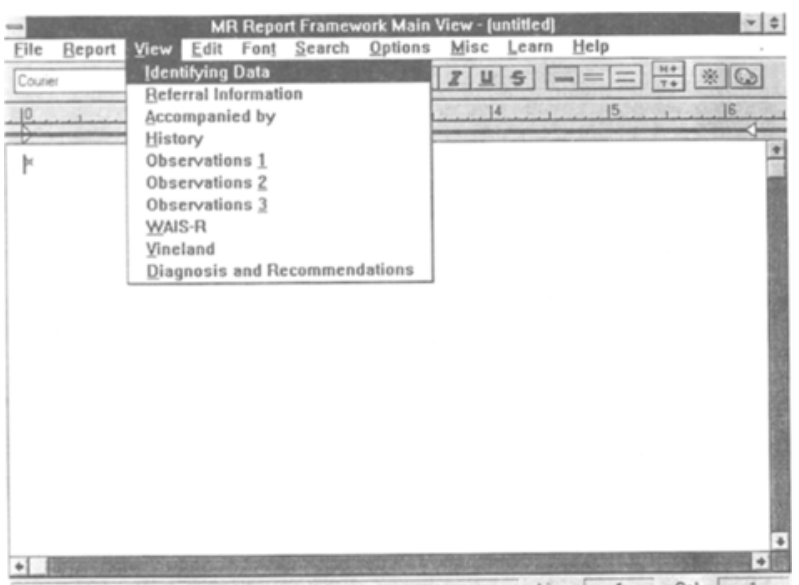

Figure 1. The Main View, with the view menu down.

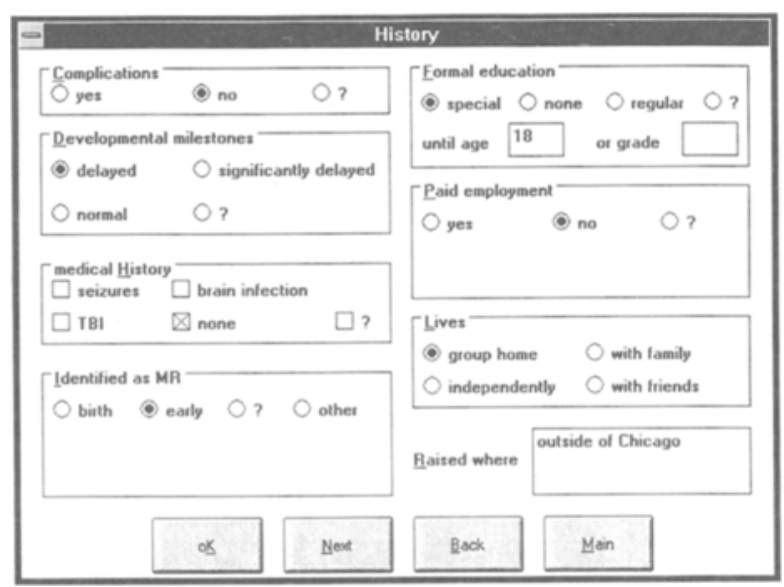

Figure 2. The History View.

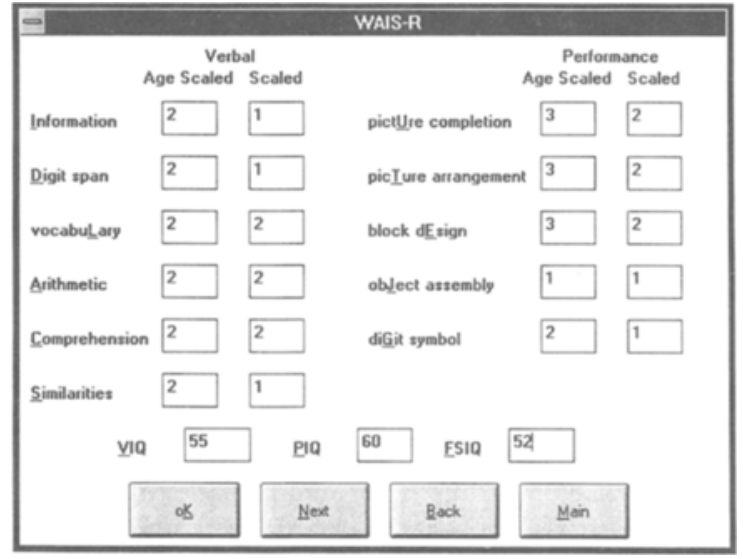

Figure 3. The WAIS-R View.

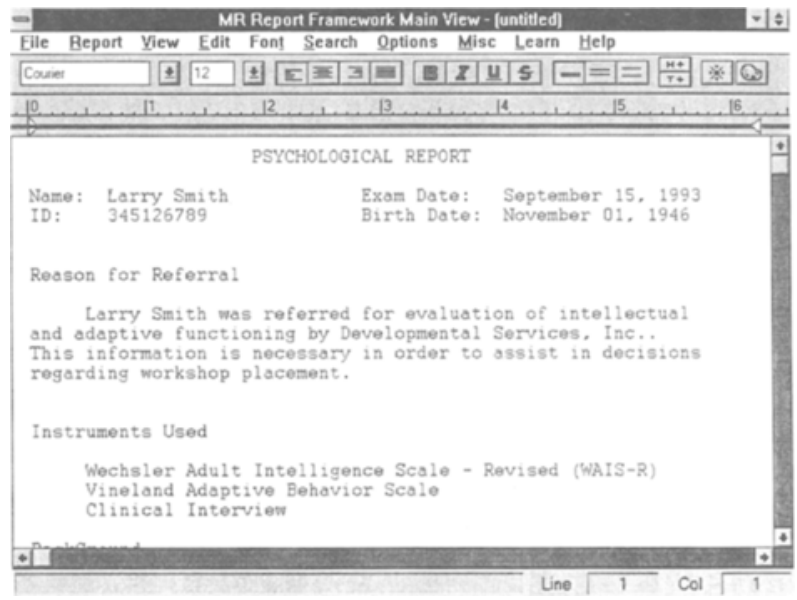

Figure 4. The Main View, with a report.

fied to use the WAIS-R and Vineland, that they are licensed at the independent-practice level in the state where they will be using the program, that the Framework will be used only under their supervision, and that the program will be used for noncommercial purposes. They should enclose a disk that is formatted for their system as well as a stamped, self-addressed disk mailer. I will copy the program onto the disk for qualified psychologists and return the disk in the mailer provided.

\section{REFERENCES}

American Psychological Association. (1986). Guidelines for computer-based tests and interpretations. Washington, DC: Author. Silverstein, A. B. (1982). Pattern analysis as simultaneous statistical inference. Journal of Consulting \& Clinical Psychology, 50, 234-240.

Sparrow, S. S., Balla, D. A., \& Cicchetti, D. V. (1984). Vineland Adaptive Behavior Scales (Interview edition; survey form manual). Circle Pines, MN: American Guidance Service.

TANNER, B. A. (1993). Computer-aided reporting of the results of mental retardation evaluations. Behavior Research Methods, Instruments, \& Computers, 25, 203-207. 